\title{
Apical Leakage of Different Gutta-Percha Techniques: Thermafil, Js Quick-Fill, Soft Core, Microseal, System B and Lateral Condensation with a Computerized Fluid Filtration Meter
}

Nimet Gençoḡlua,

Hasan Oruçoḡlub',

Dilek Helvacıoḡluc

\section{ABSTRACT}

Objectives: To investigate apical leakage of roots filled with 6 different gutta-percha techniques; Thermafil, JS Quick-Fill, Soft Core, Microseal, System B and lateral condensation using a new computerized filtration meter.

Materials and Methods: 60 extracted single rooted teeth were used. Coronal part of each tooth was removed and the root canals were prepared with step-back technique. The roots were randomly divided into six groups of 10 teeth, filled by one of the obturation technique and Kerr sealer. The teeth were kept in $100 \%$ humidity for 2 years. After this period, apical parts of roots $(10 \pm 0.05 \mathrm{~mm})$ were attached to computerized fluid filtration meter. Apical leakage quantity was determined as $\mu \mathrm{l} . \mathrm{cmH}_{2} \mathrm{O}^{-1}$. $\mathrm{min}^{-1}$.

Results: Statistical analysis indicated that Thermafil, Soft Core, Quick-Fill and System B techniques showed lower leakage than Microseal and lateral condensation $(\mathrm{P}<.05)$. However, statistically no difference was found among the core techniques and no difference was found between microseal and lateral condensation techniques ( $P>$.05).

Conclusions: Thermafil, Soft Core,Quick-Fill and System B techniques showed lower leakage than Microseal and lateral condensation. In addition, this new computerized fluid filtration meter allowed quantitative measurement of leakage easily. (Eur J Dent 2007;2:97-103)

Key Words: Fluid filtration; JS Quick-Fill; Microseal; System B; Thermafil; Lateral condensation.

\section{INTRODUCTION}

Complete obturation of the root canal system with an inert filling material and creation of a hermetic, apical seal have been proposed as goals for

$\square$ a Professor, Department of Endodontics, University of Marmara, Faculty of Dentistry, Istanbul, Turkey.

Assistant Professor, Department of Endodontics, University of Selcuk, Faculty of Dentistry,

Konya, Turkey.

Research Assistant, Department of Endodontics, University of Marmara, Faculty of Dentistry, Istanbul, Turkey.

$\square$ Corresponding author: Dr. Nimet GENCOGLU Marmara University, Faculty of Dentistry, Dept. of Endodontics Buyuk Ciftlik Sok.No:6, Nisantasi, Istanbul, Turkey.

Fax: +90 21224652 47, E-mail: ngencogluahotmail.com endodontic treatment. ${ }^{1}$ Since the most common cause of endodontic failure has been attributed to incomplete obturation, many different obturation techniques have been developed in order to increase the success of root canal treatment. Guttapercha has been material of choice for obturation since 1867. Lateral condensation has proven to be a very popular gutta-percha technique. However, its ability to conform to the internal surface of the root canal has been questioned. Brayton et $\mathrm{al}^{2}$ reported voids, spreader tracts, incomplete fusion of the gutta-percha cones and lack of surface adaptation. Also, this technique relies on sealer to fill accessory canals. Eguchi et $\mathrm{al}^{3}$ reported that lateral condensation results in excessive amounts of 
sealer and apical voids. Peters ${ }^{4}$ demonstrated that some sealer used in lateral condensation might resorb with time. This might decrease the effectiveness of the root canal obturation.

Studies have shown that softened gutta-percha can easily be moved into the canal irregularities, thus replicating the intricacies of the root canal system. There are a number of warm gutta-percha methods. These include warm lateral condensation, warm vertical condensation, coated carrier systems, injection systems and thermomechanical compaction.

The Thermafil technique introduced by Johnson in 1987 involves placing alpha phase gutta-percha on a metal carrier heating and using it to obturate the root canal. Currently used carriers are made of stainless steel, titanium or plastic. With regard to sealing of and adaptation to canal walls, Beatty et $\mathrm{l}^{5}$ reported the Thermafil technique to be superior to the lateral condensation technique. And, in an earlier study we found both thermoplasticized gutta-percha techniques Thermafil and Ultrafil to be superior to lateral condensation.6,7

Another coated carrier system, Soft Core (Soft Core System, Copenhagen, Denmark) has recently been introduced. The Soft-Core obturators consist of biocompatible plastic posts, available in ISO standard sizes and coated with thermoplastic alpha phase gutta-percha. ${ }^{8}$ The central plastic core of Soft-Core is round and hollow. Its depth corresponds to the diameter and the length of the insertion pin. However, Thermafil has a V shaped central plastic core.

JS Quick-Fill is a recently introduced mechanically thermoplasticized gutta-percha obturation method in which a titanium carrier similar to a lentulo is covered with gutta-percha and warmed by wall friction resulting from rotating it within the root canal. The plasticized gutta-percha is impulsed apically, and then the carrier can be removed from the root canal or left in place. ${ }^{9}$

Another warmed gutta-percha technique System B (endodontic heat source unit) designed to modify Schilder's technique by obturating the root canal system with a single continuous wave of thermoplasticized gutta-percha. ${ }^{6}$

Lately, Microseal system was introduced. In this technique; a master gutta-percha cone is first compacted laterally with a spreader and then thermoplasticized gutta-percha is delivered and compacted with an electric compactor in order to unite with the master cone and fill the rest of the root. $^{10}$

Fluid filtration has been used to measure microleakage since 1986 . This method has been more acceptable in measuring leakage than linear dye-penetration measurement techniques. ${ }^{11}$ The samples are not destroyed and it is possible to obtain measurements of microleakage at intervals over extended time periods. Also, dye penetration studies have limitations because trapped air has been shown to restrict the penetration of dye. ${ }^{12,13}$ It's indicated that it is more relevant to measure the amount of fluid transversing through a canal than measuring the length of a gap in a filled canal. However, conventional fluid filtration measurements are relatively subjective due to direct visual readings. A computerized, fully electronic, reliable and digital air pressure checking system is required to remove this deficiencies. ${ }^{14}$

The purpose of this in vitro study was to compare apical microleakage of teeth filled with different gutta-percha techniques using a computerized fluid filtration meter.

\section{MATERIALS AND METHODS}

Sixty extracted human non-carious single rooted teeth were used in this study. The crowns were removed and a lingual access was prepared in each tooth and instrumented using step-back technique. The teeth were irrigated with $5.25 \%$ sodium hypochloride before and after recapitulation using a size 60 master apical file and then dried with paper points. Sixty teeth were assigned to six groups (10 of each). An additional 6 teeth were used for control 3 for negative control and 3 for positive control). After air drying, a \#20 K file 2.0 $\mathrm{mm}$ longer than the working length was passed through the apex of each tooth and with the file in place three coats of nail polish were applied to external root surfaces to avoid sealing from the foramen after obturation. The negative control teeth were totally coated with three layers of nail polish, including the apical foramina. Positive controls were left unfilled.

In the first group, the teeth were obturated using the plastic carrier Thermafil (Dentsply, Maillefer, Switzerland) technique. Each canal was coated with Kerr sealer (Kerr Pulp Canal Sealer, Kerr, Romulus, MI, USAl and a size 60 Thermafil 
was heated in a Thermaprep oven for a minimum of 10 min accordance to the manufacturer's recommendations. The heated obturator was slowly inserted into the canal within $0.5 \mathrm{~mm}$ of the working length. An inverted cone bur was used to cut through the shank of each carrier.

In the second group, JS Quick-Fill obturator (JS Dental Manufacturing Inc, Ridgefeild, CT) was used in accordance to the manufacturer's instructions. No \#50 Quick-fill obturator (two size smaller than the last file used to prepare the apical third of the canal) was coated with the sealer and positioned in the canal until a slight resistance was felt. Rotation was started until gutta-percha plasticity was seen and slight pressure was applied apically until reaching working length. An inverted cone bur was used to cut through the shank of each carrier.

In the third group, the teeth were obturated with Soft-Core technique. Based on the information obtained from a "size verifier" a \#60 Soft-Core obturator was selected and heated. The root canal was coated with Kerr sealer and the plasticized Soft-Core device was inserted to the apical stop. The handle and insertion pin were removed and excess plastic core was cut away with a small inverted cone bur. ${ }^{8}$

In the fourth group, canals were obturated using the System B technique (Analytic, Sybron Dental Specialties, CA, USA) as recommended by the manufacturer. The canal walls were thinly coated with Kerr sealer and a medium-large non-standardized gutta-percha cone was placed to within $0.5 \mathrm{~mm}$. of the working length. A medium-large insert tip, which bound in the canal $3 \mathrm{~mm}$ from the working length, was used in the obturation of the canal. The System B unit was preset to $200 C^{\circ}$ during apical condensation of the primary guttapercha cone (down-pack), to $100 \mathrm{C}^{\circ}$ when adapting and condensing the apical portion of the secondary (backfill) gutta-percha cone, and finally to $250 C^{\circ}$ to soften the remainder of the secondary cone prior to vertical condensation.

In the fifth group, canals were obturated using the Microseal system (Tycom, Irvine, CA, USA). An appropriate size of master cone was selected until tug back was confirmed. The appropriate spreader was selected that was able to compact the master cone of gutta-percha $1.0 \mathrm{~mm}$ short of the working length. Finally, the additional appropriate mechanical compactors were selected, according to the manufacturer instruction. The sealer was placed into the canal and sealer coated master gutta-percha was seated. The spreader was inserted alongside the master cone at the appropriate length for compaction. Upon the withdrawal of the spreader from the canal, a tapered void had formed between the compacted gutta-percha cone and the root canal walls. The appropriate compactor was inserted in the heated gutta-percha cartridge and was coated with a uniform layer of material. The gutta-percha-coated compactor was then immediately carried to the void previously created in the canal by the spreader and was placed as close to the working length as possible, avoiding rotation as it was inserted. With the application of a resisting force to the compactor's backing-out motion, but without any apical pressure, rotation of the compactor started at a speed of $6000 \mathrm{rpm}$. After approximately 2 seconds, the compactor was removed slowly, whilst being softly pushed against one side of the canal. Rotation did not stop until the compactor was removed fully from the canal. If the canal was not completely filled, more gutta-percha was placed on the compactor. Excess gutta-percha and sealer removed from the access cavity. ${ }^{10}$

In the sixth group, the teeth were obturated with lateral condensation technique. A guttapercha master cone (Hygenic Corp, Akron, Ohio) was fitted to within $0.5 \mathrm{~mm}$ of the working length. Freshly mixed Kerr sealer was placed into the canal. A D11 spreader was inserted $1 \mathrm{~mm}$ shorter than the working length. The spreader was kept in the canal until a fine gutta-percha accessory cone was placed. The canal was considered to be adequately obturated when enough points were placed that the spreader could no longer penetrate beyond the coronal third of the canal. After complete canal obturation the coronal gutta-percha was removed with a hot instrument.

Following the obturation, the teeth were stored for 2 years at $37^{\circ} \mathrm{C}$ and $100 \%$ humidity. 2 years later, each tooth was allowed to bench cool for $1 \mathrm{~h}$ prior to testing, in order to reach room temperature.

For leakage study, apical roots of $10.00 \pm 0.05$ $\mathrm{mm}$ were sectioned using low speed diamond saw. Root sections were inserted into the plastic tube from the apical side and connected to 18-gauge stainless steel tube. The cyanoacrylate adhesive 
(Zapit, Dental Venture of America Inc., Anaheim Hills, CAl was applied circumferentially between the root and plastic tube. A computerized fluid filtration meter with a laser system used in this study have had a $25-\mu \mathrm{l}$ micropipette (Microcaps, Fisher Scientific, Philadelphia, PA, USAl mounted in horizontally. ${ }^{14} \mathrm{O}_{2}$ from a pressure tank of 120 $\mathrm{kPa}(1.2 \mathrm{~atm})$ was applied at the apical side. The pressure was constant throughout the experiment by means of a digital air pressure regulator added to pressure tank. A 25- $\mu$ l micropipette (Microcaps, Fisher Scientific, Pittsburg, PA) connected to the pressure reservoir by polyethylene tubing (Microcaps, Fisher Scientific, Pittsburg, PA). All pipettes, syringes and the plastic tubes at the apical side of the sample were filled with distilled water. Water was sucked back with the microsyringe for approximately $2 \mathrm{~mm}$. In this way, an air bubble created in the micropipette and the air bubble was adjusted to a suitable position in the syringe. The computerized fluid filtration meter was based on basically light refraction at starting and ending position of air bubble movement inside micropipette (Figure 1). Through one side

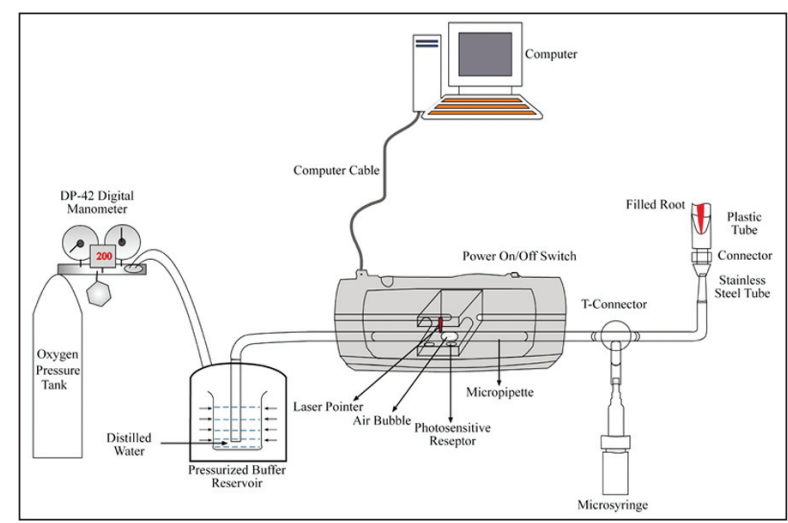

Figure 1. Computerized fluid filtration meter.

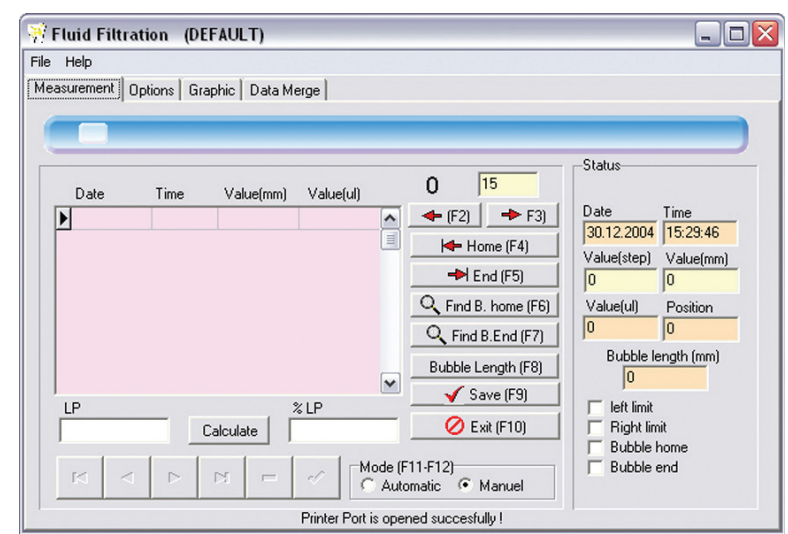

Figure 2. PC-compatible software. of the micropipette inside the device, an infrared light was passed. Two light-sensitive photodiode was arranged on the opposite side of the micropipette to detect any movement of an air bubble inside micropipette. All operations were controlled with PC-compatible software (Fluid Filtration'03, Konya, Turkey) (Figure 2). A 5-min pressurization preload of the system was completed before taking readings. Measurements of fluid movement were automatically made at 2 minutes during 8 minutes for each sample by using PC-compatible software. The software converts minute linear movement of the bubble into nanoliter movement at a rate of one measurement. This information is fed into PC-compatible software. Leakage quantity was expressed as $\mu \mathrm{l} . \mathrm{cmH}_{2} \mathrm{O} \cdot \mathrm{min}^{-1}$ and means determined.

\section{Statistical analysis}

All data were statistically analyzed by One-way ANOVA followed by Duncan tests. The confidence level was used $95 \%(P<.05)$.

\section{RESULTS}

Mean microleakage measurements and standard errors, in $\mu \mathrm{l} . \mathrm{cmH}_{2} \mathrm{O}^{-1} \cdot \mathrm{min}^{-1} 1.2 \mathrm{~atm}$, are shown in Table 1.

According to the results of this study, Microseal technique showed the most leakage followed by lateral condensation technique $(\mathrm{P}<.05)$ and both techniques showed more leakage than other techniques (Thermafil, Soft Core, Quick-fill, System

Table 1. Mean leakage values $\left(\mu \mathrm{l} . \mathrm{cmH}_{2} \mathrm{O}^{-1} \cdot \mathrm{min}^{-1} \mathrm{x}\right.$ $10^{-4}$ ) and standard deviation (SD) for each tested technique.

\begin{tabular}{lc}
\hline Groups & Mean Value \pm SD \\
\hline Thermafil & $0.26 \pm 0.08$ \\
Quick-Fill & $0.32 \pm 0.08$ \\
Soft Core & $0.30 \pm 0.06$ \\
System B & $0.28 \pm 0.07$ \\
Microseal & $0.50 \pm 0.05$ \\
Lateral Condensation & $0.44 \pm 0.10$ \\
Positive Control & $52.5 \pm 0.10$ \\
Negative Control & $0.00 \pm 0.00$ \\
\hline
\end{tabular}


B) $(P<.05)$. However, no difference was found between Microseal and lateral condensation techniques ( $P>$.05). Although Thermafil showed the least leakage statistically the difference was not significant among Thermafil, Quick-Fill, Soft Core and System B.

The positive controls demonstrated extreme amounts of apical leakage. Mean leakage for the positive controls was observed to be $52.5 \pm 0.10$ $\mu \mathrm{l} . \mathrm{cmH}_{2} \mathrm{O}^{-1}$. min-1.10-4 $1.2 \mathrm{~atm}$. The negative controls registered no detectable bubble movement at $1.2 \mathrm{~atm}$, for a minimum of $5 \mathrm{~min}$ before beginning of each data collection.

\section{DISCUSSION}

In this in vitro study, different obturation techniques' apical leakage was tested using a new computerized fluid filtration meter. In vitro dye leakage tests are very popular in literature, because of easy use and cheap laboratory processes. But recently, because of the high number of appliance variations, contradictory and non-comparable comments, entrapped air phenomena and the reliability of dye leakage tests are questioned. ${ }^{15}$ One of the major problem related to the dye penetration method is that entrapped air in a void along a root canal filling may hinder dye penetration. ${ }^{16}$ Goldman et $\mathrm{al}^{12}$ demonstrated the usefulness of dye penetration method at their experiment with unfilled root canals. From that point of view, fluid filtration microleakage test is supposed to be a better alternative than dye leakage tests for determining the leakage. The pressure applied in this method helps to eliminate the entrapped air in root-canal fillings. The additional advantages of fluid filtration can be listed as: the measurements can be repeated at various time periods, the exact time that the maximum leakage occurs can be determined; the samples are not affected from the test procedures. Also, by fluid filtration test, trough and trough evaluation of the leakage pattern can be made. Dye leakage test does not include the whole length of the root-canal fillings. Such a full-length observation can be stimulated clinical leakage and infection caused by leakage models.

The computerized fluid filtration meter used in this study has some advantages over the conventional ones with computer controlling and digital air pressure arrangement. Additionally, the move- ment of a small air bubble can be observed by laser diodes computer controlled rather than visual following.

According to this study, the core (Thermafil, Quick-Fill and Soft Core) techniques and System B showed better sealing properties than Microseal and lateral condensation techniques.

Beatty et $\mathrm{al}^{5}$ and Dummer et $\mathrm{al}^{17}$ also found the Thermafil technique to result in less leakage than did the lateral condensation technique. In our previous dye leakage study, Thermafil QuickFill core techniques were found to be superior to lateral condensation technique. ${ }^{18}$ Pommel and Champs ${ }^{19}$ investigated apical sealing of single-cone, lateral condensation, vertical condensation, Thermafil and System B obturation techniques by using fluid filtration microleakage system taking measurements at 24 hours and 1 month. They found that the single cone technique produced the most apical leakage in 24 hours. They indicated that this result was attributable to the greater volume of sealer required for the single cone technique. After 1 month, they found that the Thermafil, System $B$ and vertical condensation techniques produced less leakage than did the two other techniques. The lateral condensation showed more apical leakage after 1 month, whereas the single cone technique produced the greatest leakage. It seemed that the use of a larger volume of sealer results in shrinkage more often than does the use of a small volume, as was the case with the cone and lateral condensation techniques showed much more leakage than the compaction technique. Kontakiotis et $\mathrm{al}^{20}$ investigated long term sealing ability of different sealers and found more leakage after 2 years storage in water than before storage. In the present study, the specimens were kept in humidity for 2 years to create the most microleakage.

The reduced ratio of sealer to gutta-percha may improve the long term seal provided by root canal fillings. Endodontic sealers are soluble materials and the shrinkage may result in potential leakage pathways in root canal fillings. ${ }^{21,22} \mathrm{Wu}$ et $\mathrm{al}^{23} \mathrm{re}$ ported that gross amounts of sealer may result in increasing amount of leakage. Gencoglu ${ }^{24}$ evaluated the sealer-gutta-percha ratio in Thermafil, Quick-Fill, Soft Core Microseal System B and lateral condensation techniques. They found that Thermafil, JS Quick-Fill and Soft Core techniques had more gutta-percha content than Microseal, 
System B and lateral condensation techniques. They stated that higher sealer content might lead to higher leakage amounts. In the present study, Microseal and lateral condensation techniques showed higher leakage results in 2 years. Also, Cathro and Love ${ }^{25}$ found that Microseal technique produced a heterogeneous fill, but System B/Obtura II technique produced a homogenous fill at all levels of the root canal filling.

On the other hand, Silver et $\mathrm{al}^{26}$ compared two vertical condensation techniques Touch'n Heat modified and System B and they found System B produced acceptable obturation. Kytridou et $\mathrm{al}^{27}$ investigated adaptation and short and long term seal ability of Thermafil and System B and found that both obturation techniques produced substantial filling material movement in the canal irregularities. On the other hand, although no difference were detected between Thermafil and System B, in teeth which were immersed in Indiana ink for 24 hours or 10 days, significantly more leakage was detected in Thermafil group which were immersed 67 days in HBS and 90 hours in dye solution. Shakespeare and Donnelly, ${ }^{28}$ and Guabivala et al $^{29}$ found less leakage due to lateral condensation than to the JS Quick-Fill technique whereas Pallares and Faus ${ }^{30}$, Canalda-Sahli et $\mathrm{al}^{31}$ found no statistical difference between them. Davalou et al $^{32}$ compared sealing ability of System B and Microseal techniques and found statistically no difference. Pomel and Camps ${ }^{19}$ investigated sealing ability of Thermafil, warm vertical condensation technique and single cone technique by using fluid filtration, electrochemical and dye leakage methods. They found no correlation among the results obtained with the three methods of evaluation. This result corroborates to other studies that also attempted to compare different microleakage methods. ${ }^{33-37}$ Therefore, more research should be done about the methodology of leakage tests and standardization is needed for test protocols. So far, fluid filtration method seems the best method for evaluation microleakage for the time being and a computerized fluid meter has some advantages over the conventional method. Even though with these in vitro results, in-vivo apical leakage cannot be determined. Further clinical studies are necessary to confirm these results.

\section{CONCLUSIONS}

Thermafil, Soft Core,Quick-Fill and System B techniques showed lower leakage than Microseal and lateral condensation. In addition, this new computerized fluid filtration meter allowed quantitative measurement of leakage easily.

\section{REFERENCES}

1. Nyguen NT. Obturation of the root canal system. In: Cohens S, Burns, RC, editors. Pathways of the pulp. 3rd ed. St. Louis: CV Mosby Co, 1984.

2. Brayton SM, Davis SR, Goldman M. Gutta-percha root canal fillings. An in vitro analysis. I. Oral Surg Oral Med Oral Pathol 1973;35:226-231.

3. Eguchi DS, Peters DD, Hollinger JO, Lorton L. A comparison of the area of the canal space occupied by gutta-percha following four gutta-percha obturation techniques using Procosol sealer. J Endod 1985;11:166-175.

4. Peters DD. Two-year in vitro solubility evaluation of four Gutta-percha sealer obturation techniques. $J$ Endod 1986;12:139-145.

5. Beatty RG, Baker PS, Haddix J, Hart F. The efficacy of four root canal obturation techniques in preventing apical dye penetration. J Am Dent Assoc 1989;119:633-637.

6. Gencoglu N, Samani S, Gunday M. Dentinal wall adaptation of thermoplasticized gutta-percha in the absence or presence of smear layer: a scanning electron microscopic study. J Endod 1993;19:558-562.

7. Gencoglu N, Samani S, Gunday M. Evaluation of sealing properties of Thermafil and Ultrafil techniques in the absence or presence of smear layer. J Endod 1993;19:599603.

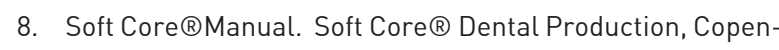
hagen K, Denmark.

9. JS Quick-Fill Instructions. JS Dental Manifacturing Inc. Ridgefield, CT, USA.

10. Korzen BH. Endodontic obturation using the MicroSeal technique. Oral Health 1997;87:67-70;73-74.

11. Antonopoulos KG, Attin T, Hellwig E. Evaluation of the apical seal of root canal fillings with different methods. $J$ Endod 1988;24:655-658.

12. Goldman M, Simmonds S, Rush R. The usefulness of dyepenetration studies reexamined. Oral Surg Oral Med Oral Pathol 1989;67:327-332.

13. Spangberg LS, Acierno TG, Yongbum Cha B. Influence of entrapped air on the accuracy of leakage studies using dye penetration methods. J Endod 1989;15:548-551.

14. Orucoglu H, Sengun A, Yilmaz N. Apical leakage of resin 
based root canal sealers with a new computerized fluid filtration meter. J Endod 2005;31:886-890.

15. Wu MK, Wesselink PR. Endodontic leakage studies reconsidered. Part I. Methodology, application and relevance. Int Endod J 1993;26:37-43.

16. Wu MK, De Gee AJ, Wesselink PR. Fluid transport and dye penetration along root canal fillings. Int Endod $J$ 1994;27:233-238.

17. Dummer PM, Lyle L, Rawle J, Kennedy JK. A laboratory study of root fillings in teeth obturated by lateral condensation of gutta-percha or Thermafil obturators. Int Endod $J$ 1994;27:32-38.

18. Gencoglu N, Garip Y, Bas M, Samani S. Comparison of different gutta-percha root filling techniques: Thermafil, Quick-fill, System B, and lateral condensation. Oral Surg Oral Med Oral Pathol Oral Radiol Endod 2002;93:333-336.

19. Pommel L, Camps J. In vitro apical leakage of system B compared with other filling techniques. $J$ Endod 2001;27:449-451.

20. Kontakiotis EG, Wu MK, Wesselink PR. Effect of sealer thickness on long-term sealing ability: a 2-year follow-up study. Int Endod J 1997;30:307-312.

21. Langeland K. Root canal sealants and pastes. Dent Clin North Am 1974;18:309-327.

22. Schilder H. Filling root canals in three dimensions. Dent Clin North Am 1967;11:723-744.

23. Wu MK, van der Sluis LW, Wesselink PR. A preliminary study of the percentage of gutta-percha-filled area in the apical canal filled with vertically compacted warm guttapercha. Int Endod J 2002;35:527-535.

24. Gencoglu N. Comparison of 6 different gutta-percha techniques (part II): Thermafil, JS Quick-Fill, Soft Core, Microseal, System B, and lateral condensation. Oral Surg Oral Med Oral Pathol Oral Radiol Endod 2003;96:91-95.

25. Cathro PR, Love RM. Comparison of MicroSeal and System B/Obtura II obturation techniques. Int Endod J 2003;36:876882.

26. Silver GK, Love RM, Purton DG. Comparison of two vertical condensation obturation techniques: Touch ' $\mathrm{n}$ Heat modified and System B. Int Endod J 1999;32:287-295.

27. Kytridou V, Gutmann JL, Nunn MH. Adaptation and sealability of two contemporary obturation techniques in the absence of the dentinal smear layer. Int Endod J 1999;32:464474.

28. Shakespeare RC, Donnelly JC. An in vitro comparison of apical microleakage after obturation with JS Quick-Fill or lateral condensation. J Endod 1997;23:312-314.

29. Gulabivala K, Holt R, Long B. An in vitro comparison of thermoplasticised gutta-percha obturation techniques with cold lateral condensation. Endod Dent Traumatol
1998;14:262-269.

30. Pallares A, Faus V. A comparative study of the sealing ability of two root canal obturation techniques. $J$ Endod 1995;21:449-450.

31. Canalda-Sahli C, Berastegui-Jimeno E, Brau-Aguade E. Apical sealing using two thermoplasticized gutta-percha techniques compared with lateral condensation. $J$ Endod 1997;23:636-638.

32. Davalou S, Gutmann JL, Nunn MH. Assessment of apical and coronal root canal seals using contemporary endodontic obturation and restorative materials and techniques. Int Endod J 1999;32:388-396.

33. Barthel CR, Moshonov J, Shuping G, Orstavik D. Bacterial leakage versus dye leakage in obturated root canals. Int Endod $J$ 1999;32:370-375.

34. Camps J, Pashley D. Reliability of the dye penetration studies. J Endod 2003;29:592-594.

35. Delivanis PD, Chapman KA. Comparison and reliability of techniques for measuring leakage and marginal penetration. Oral Surg Oral Med Oral Pathol 1982;53:410-416.

36. Matloff IR, Jensen JR, Singer L, Tabibi A. A comparison of methods used in root canal sealability studies. Oral Surg Oral Med Oral Pathol 1982;53:203-208.

37. Pommel L, Jacquot B, Camps J. Lack of correlation among three methods for evaluation of apical leakage. $J$ Endod 2001;27:347-350. 